\title{
ABC-PSO OPTIMIZATION TECHNIQUE FOR PARAMETER OPTIMIZATION IN CIRCULAR LOG PERIODIC NANO ANTENNA
}

\author{
Sathisha $\mathbf{T}^{\mathbf{1}}$, Ananda Kumari $\mathbf{R}^{\mathbf{2}}$ \\ ${ }^{1}$ Research Scholar, Electronics, Tumkur University, Karnataka, India \\ ${ }^{2}$ Head \& Associated Professor, Sree Siddaganga College of Arts Science and Commerce for Women, Karnataka, India
}

\begin{abstract}
It is applicable to note that, in the overall wireless communication mechanism, the role played by the antenna is beyond the realm of words. In fact, the deployment of the antenna the wireless network faces the grave challenges of the communication of the high speed signals. Of late, the Nano_antennas make their elegant presence in several spheres of the technology. However, it is highly essential, at present, to have an overall awareness for the purpose of a balanced design of nano_antennas with broadband feedbacks. These days, many a technique is doing its elegant rounds invested in examining the spectral features of the log periodic nano_antenna by the excitation method of reception and discharge. The field improvement of nano_antenna can be harmonized by several geometric, constraints, including the outer radius, the tooth angle, and the ratio of the radial sizes of consecutive teeth, which furnish effective check on the spectral resonance position and the field renovation. Taking the challenges in to consideration, a novel hybrid ABCO-PSO optimization approach is launched in the document, which involves a gifted prospect for planning and adapting the LPNA. With the result, the adaption of the related constraints incredibly increases the effectiveness of the mechanism to envision it to an acceptable level. The design of the LPNA is made by adapting the constraints which is highly appropriate for the wireless communication which is assessed for field improvement and the Purcell factor of Conventional Log periodic Toothed Antenna and Log periodic Toothed Circular Nano Antenna.
\end{abstract}

Keywords: Circular Log Periodic Nano Antenna, Field Enhancement, Purcell Factor, Genetic Algorithm, Artificial Bee Colony.

\section{INTRODUCTION}

Of late, the design of compact and cost-conscious antennas to support diverse benchmarks concurrently for the wireless devices has emerged as a fascinating research subject among the industrial magnates and also the academic cream. The upcoming antennas have to be well-geared to shore up the multiband tasks, illustrate insignificant shape features, facilitate easy integration with the RF circuits, and, the most significant feature being their cost-effectiveness [1]. The concept of employing antennas to obtain solar energy was initially envisaged Robert L. Bailey in the year 1972 [2]. The incredible impact of the geometry on field improvement and the Purcell factor were methodically defined and experimented [1]. Further, the multi-resonant field enhancement was appraised extensive by means of the conformal modification.

The antennas are extensively employed at the radio and microwave frequencies to couple/emit energy from a source to free space with a far-field discharge of condensed angular divergence. In view of the reciprocity, they also gather radiation effectively from the defined directions. With the onset of the nanotechnology, the antennas functioning at the optical/near-infrared frequencies have become handy, excelling the energy localization skills of the conventional optical segments like the mirrors and lenses [3].In fact, the Nano antennas are habitually employed as the electromagnetic collectors. The Nano antenna electromagnetic collector (NEC) is targeted at the midinfrared wavelengths, where the traditional photovoltaic (PV) solar cells are found to be ineffectual and especially where the solar energy is abundantly available. The notion of devising the NEC is dependent on the scaling of radio frequency antenna theory [4].The optical response of the Plasmonic metal nano particles is well-defined by the classical electro dynamics. The Plasmonic response is offered by a complex permittivity with a negative real part which originates from the retarded coherent collective surface oscillations of electrons [5].

The log-periodic dipole array (LPDA) antenna represents one of the aspirants for the modern wireless communications mechanism. The design technique and the accomplishment of the traditional LPDA antenna has been recognized long back right from the 1960s. Theefficiency in execution of the LPDA antennas can be improved by means of the clamped-mode technique. The clamped-mode conventional LPDA antenna, in turn, needs only half the number of dipole elements and $\mathrm{d}$ feed lines [6]. The Logarithmic periodic antenna constitutes a scientific instrument for the power spectrum radio data surveillance. It plays a vital part in the effective identification of the solar radiation as it furnishes the sensitive segment of the radio detector [7]. Such kind of antenna is effectively employed as the fundamental receiving segment. Classically, the radiation of the quantum emitters, like the quantum dots or colour centres in nano diamonds can be robustly enhanced 
and highly directed by coupling an emitter to the optical nano antennas. Based on the concept of reciprocity, the corresponding type of antennas are competent to efficiently locate an inward radiation [8]. A swift state initialization speed, quicker than the quantum state coherence rate, is also needed for consistent quantum computation functions [9].Single antenna nanostructures illustrate the dichroic conduct in the far-field spectrum, based on the polarization of the intruding light [10].

The antennas may be extensively employed in the domain of the microscopy, spectroscopy, data-communication, including the solar energy harvesting. It is recognized that the antennas can be produced with a metal or a dielectric material [2]. The nano antenna arrays may be so devised as to absorb any frequency of light [8]. The resonant frequency of a nano antenna may be chosen by modifying its length. Even though the nano antennas gleamin their sheen with glittering gains, further upcoming sophistications are the need of the hour so as to efficiently to execute the related antennas at superior frequencies to avert it from becoming impractical. The existing nano antennas are generated by means of the electron beam (e-beam) lithography. However, it is unfortunate that the corresponding process is sluggish, thereby becoming highly costly.

\section{RELATED WORK}

P Jafarlou et.al [11] fantastically launched an innovative fast analysis technique for a new terahertz waveguide for photomixing. The wave-guiding mixer configuration was adapted by the parallel plate waveguide (PPWG) in which the top plate was substituted by means of a recurring assortment of sub-wavelength nano-slits. The substrate of the PPWG was built up with a swift photoconductive material in which laser photo mixing/absorption took place. The typical equation of the adaptive PPWG was employed as a THz waveguide and was obtained systematically, and its guided modes were extensively investigated over the $\mathrm{THz}$ range of frequencies.

M. C. Marcon[12], carried out a number of researches regarding the two- and three-dimensional frameworks with the submicron dimensions, like the photonic crystals, patterned magnetic media, micro- and nanoelectromechanical systems (MEMs/ NEMs), microfluidics chips, and so on which were ever-present in numerous applications cutting across several exploration avenues. The paramount need for engineering these tools has motivated the growth of the innovative nanofabrication and nanopatterning techniques. He discussed in detail the vital contributions in the laser-based nano-patterning and nanofabrication approaches. Specifically, the focus was on the recently designed nano-patterning approaches were dependent on the employment of the incredible ultraviolet laser light which contributed a lot to the quick and effective fabrication of the recurrent nano-structures.

Guohua zhai et.al[13], invited world-wide attention by effectively launching various parasitic dipole elements served as director cell which were supplemented to the smaller end of the printed Log-periodic dipole array (PLPDA) antenna for the augmentation in advantages. The antenna was primarily dependent on the integration of the PLPDA antenna and the quasi-Yagi antenna approaches, and also the printed Log-Yagi dipole array antenna (PLYDA). The number and the distribution of the director cell were deeply debated and adapted. A model PLYDA antenna furnished by means of the half mode substrate integrated waveguide (HMSIW) was created, together with an HMSIW PLPDA antenna for the purpose of assessment and contrast.

Wang et.al [14] proficiently propounded a 30 log-periods microstrip angular log-periodic meander-line SWS. The dispersion of such a type of the SWS was found to be feeble, which illustrated the fact that it was competent to function in an extensive operating bandwidth. The glittering gain was that the functional voltage was incredible lesser than that of the tradition TWT at the identical functional frequency and the geometrical dimension was found to be extremely smaller than that of the traditional TWT. The beam-wave interaction of the angular log-periodic meander-line TWT was estimated by means of the particle-in-cell approach.

\subsection{Problem Definition}

Over the last few years, the nano antenna designs have succeeded in annexing a vital position in the wireless mechanisms thanks to the vital part played by the antenna in the domain of the entire communication system. In view of the sophisticated growth in the technology and zooming requirements in the wireless communication the upcoming technology is badly in need of a single antenna to support various allotted frequency bands as the capacity continues to one of the thorny issues in the wireless networks. In the literature, review a host of optimization techniques are reviewed which are efficiently employed to tackle the daunting challenges related to the design of nano antenna by adapting the constraints. However, it is unfortunate that when a titanic number of antenna and design variables are employed, it becomes humanly impossible to effectively and efficiently evaluate their attributes. In the novel technique, a renovated algorithm and gifted technique which adapt the dimension and intricacy of the antenna are envisaged.

\section{PROPOSED METHOD FOR PARAMETER} OPTIMIZATION IN LOG PERIODIC NANOANTENNA USING HYBRID ABC-PSO.

\subsection{Overview}

The Nano-Antennas play a very significant part in plentiful spheres of technology. However, it is highly essential to have an improved comprehension for the objective design of the nano-antennas with broadband responses. Even though, there is a host of techniques which are employed to investigate the spectral attributes of the log periodic nano antenna by the excitation method of reception and emission. It becomes humanly impossible to evaluate their attributes effectively, when large numbers of antenna and design 
variables are employed, Hence, with an eye to dynamically evaluate and optimize the design parameters, the proposed technique is developed. Here, the Purcell factor and the field enhancement are analyzed for the conventional log periodic nano antenna as well as the log periodic toothed circular nano antenna. The Field Enhancement of the Nano antenna can be adapted by means of the geometric constraints like the Outer radius, the Tooth angle, and the ratio of the Radial sizes of successive teeth, which furnish a significant control over both the spectral resonance position and the field enhancement with the Purcell factor. Accordingly, in the document, a novel hybrid optimization method is flagged off to adapt the constraints. The added feature to our proposed technique is that the hybridization of the optimization algorithms likes the $\mathrm{ABC}$ and PSO.

\subsection{Nano Antenna Structure}

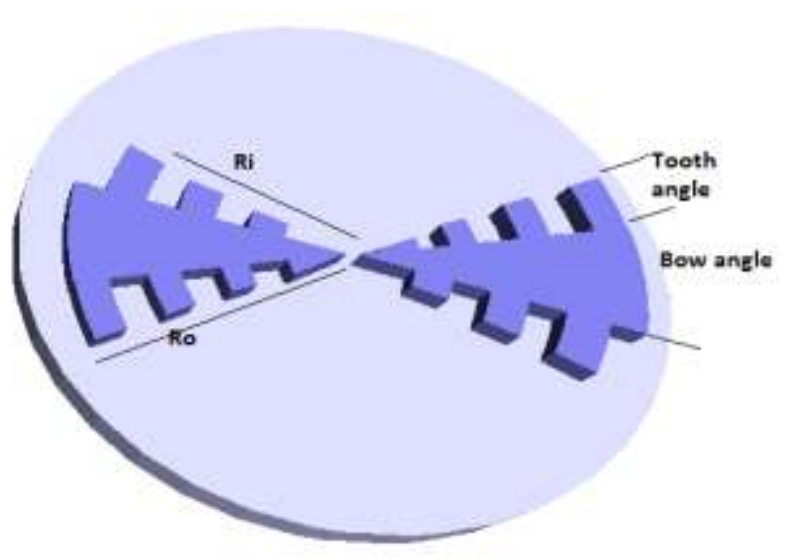

Fig 3.1 Structure of Nano antenna

\subsubsection{Nano Antenna Geometry}

The nano antenna framework is characterized by the constraints shown below.

- $\quad$ The Outer Radius $\left(R_{o}\right)$

- $\quad$ The Inner Radius $\left(R_{i}\right)$

- Tooth angle $(\delta)$

- $\operatorname{Bow}$ angle $(\chi)$

- Number of teeth $\left(t_{n}\right)$

- $\quad$ Thickness $(T)$

- Gap between the two $\operatorname{arms}\left(G_{a}\right)$

The radial size of the successive teeth is represented by means of Equation 1 shown hereunder.

$\eta=\frac{R_{o}}{R_{o+1}}$

To authenticate the optical response of LPNA, a renovated drude dielectric field is employed as represented in the following Equation 2

$$
\xi_{u}=\xi_{\infty}-\frac{u_{m}^{2}}{u^{2}+j u \gamma}
$$

Where

$\xi_{\infty}, u_{m}$ and $1 / \gamma$ representing the Dielectric constant and in the case of silver, the constraints employed are shown below.

$\xi_{\infty}=4.039, u_{m}=9.154$ and $\gamma=0.815 e V$

\subsubsection{Purcell Factor $\left(P_{f}\right)$}

The Purcell factor $\left(P_{f}\right)$ is evaluated by means of the following Equation 3.

$P_{f}=\frac{\iint P_{r}(\theta, \phi) d \Lambda}{\iint P_{o}(\theta, \phi) d \Lambda}$

$\iint P_{r}(\theta, \phi) d \Lambda$ and $\iint P_{o}(\theta, \phi) d \Lambda$ represent the power radiating to the far field to the innovative technique.

\subsubsection{Field Enhancement}

The spectrum attributes of field enhancement $\left(E / \mathrm{E}_{\text {in }}\right)$ in which the Nano-antenna is tempted by a normal incident plane-wave polarized in the y direction , and the Nano antenna is also simulated by an electric field emitter placed at the gap equidistantly between the two halves of the LPTCNA to evaluate the Purcell factor attributes. The electric dipole is situated in the identical plane as the antenna and has been oriented in its longitudinal direction. The field enhancement is evaluated as per Equation 4 given below.

$$
E / \mathrm{E}_{\text {in }}
$$

The effective resonance wavelength of the traditional log periodic toothed antenna is estimated by means of Equation 5 shown below.

$$
\tau_{e f f}=R_{o}(1+\sqrt{\eta})(\chi+\delta) \pi / 180^{\circ}
$$

The effective resonance wavelength of log periodic toothed circular nano antenna is calculated with the help of the following Equation 6.

$$
\tau_{\text {eff }}=\left(R_{o}+R_{i}\right)(\chi+\delta) \pi / 180^{\circ}
$$

\subsection{Hybrid Swarm Intelligent Based Optimization}

The field enhancement and Purcell factor of the nano antenna are generally adapted by means of the geometric constraints like the outer radius, the tooth angle, and the 
ratio of the radial sizes of successive teeth, which have an effective control over both the spectral esonance position, is achieved by means of the optimization technique. It is observed that the distinctive performances of the PSO and $\mathrm{ABC}$ are excelled by the hybrid ABC-PSO, where, the position of a food source characterizes a potential solution of the optimization issues and the arbitrary value of fitness of the related solution. The number of the PSO optimal values or the onlooker bees is equivalent to the number of solutions in the population.

\subsubsection{Artificial Bee Colony}

When the initialization is completed, the population of the positions is subjected to repetitive cycles of the investigation procedures of the employed bees, the onlooker bees, and the scout bees. Now, the onlookers and employed bees perform the utilization procedure in the search space and the scouts effectively regulate the exploration procedure and fine-tune the solution.

\subsubsection{Particle Swarm Optimization}

When the best fitness function is selected, the new population is produced and thus the new populations are created for the purpose of locating the best fit expressions among the other expressions in the population. The new populations are estimated by setting two specifications defined by the PSO technique.

\subsubsection{Fitness Function}

Every optimization program is restricted by certain fitness functions, and depending upon the nature and behaviour of the inputs and outputs, the fitness functions also undergoes alterations. The value produced from the fitness function is known as the fitness value. In the document, the population, which is defined earlier, is considered for the evaluation of the fitness. Every expression in the population is chosen for estimating its fitness. Thereafter, the upshot is processed with the help of the values mentioned above. Depending upon those values, the accuracy and the fitness values are evaluated. In an identical manner, every expression is treated and the fitness values of all the expressions are chosen. The relative task continues until the specific iteration is reached. As regards the PSO, the number of iterations is furnished invariably by the user. The expression with maximum fitness value is shortlisted as the global best.

\subsubsection{Updating p Best and g Best}

The specifications represent the position and velocity of the elements in the population, in the existing environment, and the expressions are deemed as the segments of the population. Subsequently, for the purpose of attaining the new populations, the velocity and the position of each value are revised. Each particle is aware of its best value till now ( $\mathrm{p}$ best) and its position. In addition, each particle has sufficient knowledge on the best value till now in the group ( $g$ best) among the $p$ bests. The related data represents the correlation of knowledge regarding the quality or excellence of the performance of the neighboring particles. The procedure is repeated till a termination condition is satisfied and in a large majority of the cases, iterations are taken as the termination criteria.

\section{Steps in Multilevel ABC-PSO}

Step 1: The ABC produces an arbitrary initial population as illustrated in the following Equation (7) where P represents the variables and $\boldsymbol{P}_{\boldsymbol{i}}$ denotes a vector value. Here, $i$ correspond to the Number of optimized parameters.

$$
P_{i}^{\min }<P_{i}<P_{i}^{\max }
$$

Step 2: Estimate the fitness value for PSO optimization and if it is superior to the best fitness value, Set current value as the new Best

Step3: Evaluate velocity of particle and position update in accordance with the following Equations 8 and 9. Here $V_{p}$ signifies the velocity, $V_{\max }$ denotes the maximum velocity, $\mathrm{r}$ represents an arbitrary value and y correspond to the current position

$$
\begin{aligned}
V_{p i} & =V_{p i}+c_{1} r_{1}\left(q_{i}-y_{i}\right)+c_{2} r_{2}\left(q_{g}-y_{i}\right) \\
y_{i} & =y_{i}+v_{p i}
\end{aligned}
$$

Step 4: Allocate the Onlooker bee to the Employed to pbest solution depending upon the probabilities.

Step 5: If fit (Best Onlooker) < p (best), then substitute, the best onlooker with the corresponding pbest Neighborhood value.

Step 6: An onlooker bee selects the optimized best value related to the corresponding food source and the fitness function is evaluated. Here $f_{i}$ represents fitness function and fit $_{i}$ indicates the fitness after a transformation.

$$
f_{i t}=\frac{1}{f_{i}}
$$

Step 7: The criterion is terminated when the maximum Iteration is reached.

\section{Ranking of ideal Solution}

In the priority based categorization of the ideal solution, the Merging and Classification is performed by selecting the ideal values from the multi-level ABC-PSO. Thus the best fitness is classified as the solution. The priority based classification of the ideal solution is envisaged so as to grade the best ideal solution. At the outset, the function of merging the ideal solution is carried out as illustrated in Equation 11 shown below. 


$$
R_{s}=\{A B C\} \bigcup\{P S O\}
$$

Where,

$R_{s}$ - refers to the Best Solution

$A B C-$ Represents the generated best solution employing the $A B C$

PSO - Signifies the generated best solution utilizing the PSO

\section{Ultimate Solution}

After integrating all the solutions, the solution is classified to arrive at the Ultimate ideal solution. Thus these values are deemed as the finest solution.

\section{EXPERIMENTAL SETUP}

The Parameter Optimization in the Log Periodic NanoAntenna is carried out in the working platform of MATLAB (version 7.11) with machine configuration shown as follows. Processor: Intel core i5

CPU Speed: $3.20 \mathrm{GHz}$

OS: Windows 7

RAM: 4GB

\subsection{Results and Discussion}

The design parameter optimization of the log periodic Nano antenna is effectively exhibited in the ensuing debate. Here, the parameters are optimized by means of the Hybrid optimization method like the ABC-PSO and it is assessed and contrasted with other distinct modern methods like the $\mathrm{ABC}$, PSO and GA. The appraisal is performance in accordance with the impact of the field enhancement and Purcell factor of the Nano antenna. The antenna parameter optimization is illustrated as per the conventional log periodic nano antenna and the log periodic toothed circular nano antenna. The outer radius of the circular-toothed structure on the field enhancement and the Purcell factor of LPNA are tentatively examined and thereafter processed. The Log periodic nano antenna is invariably supported by a silica substrate having the permittivity $\ell_{\text {sub }}=2.25$ with Thickness $(T=90 \mathrm{~nm})$ and radius $(r=583 \mathrm{~nm})$

Table 1 Field Enhancement of Conventional Log Periodic Tooth Antenna for different values

\section{Field Enhancement of Conventional Log Periodic Tooth Antenna}

\begin{tabular}{|l|l|l|l|l|}
\hline & $\begin{array}{l}\text { Proposed } \\
\text { Technique }\end{array}$ & \multicolumn{3}{|l|}{ Existing Methods } \\
\hline Iteration & ABC-PSO & GA & ABC & PSO \\
\hline 50 & 1.2 & 10.8 & 12.9 & 5.9 \\
\hline 75 & 1.3 & 17.5 & 18.3 & 6.8 \\
\hline 100 & 3.7 & 36.5 & 3.5 & 5.5 \\
\hline
\end{tabular}

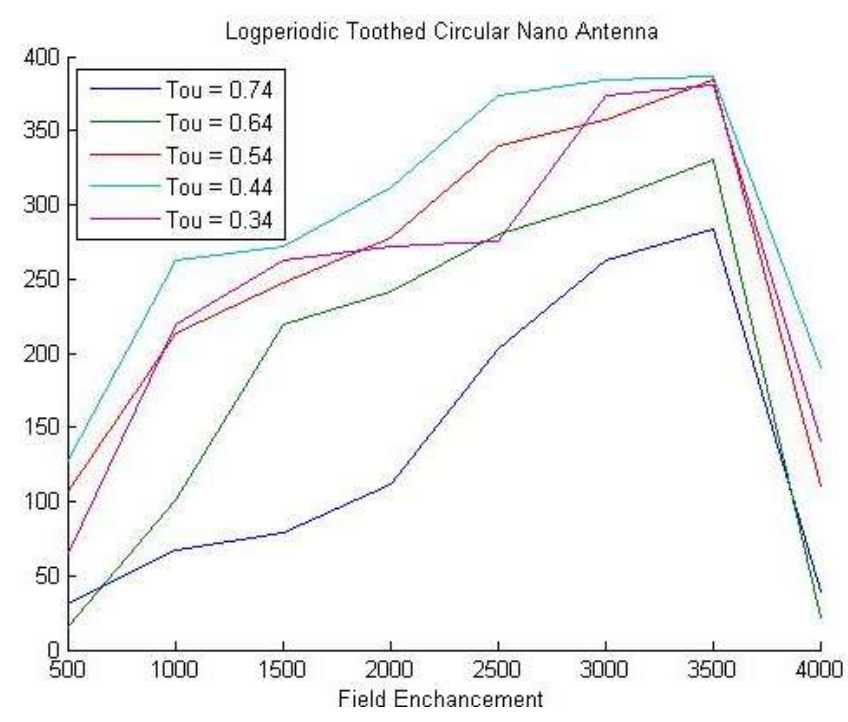

Fig 4.1 Field Enhancement of Log periodic Toothed Circular Nano Antenna for different $T_{o u}$ values

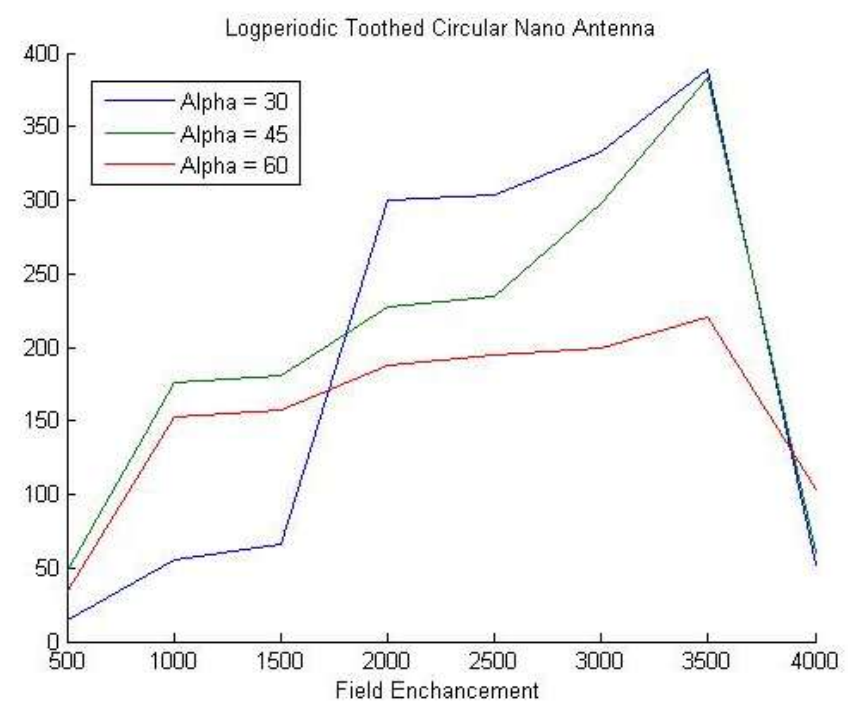

Fig 4.2 Field Enhancement of Conventional Log Periodic Tooth Antenna for Alpha=30, 45 and 60

* The significant impact of the ratio of the radial size of consecutive teeth on the field enhancement with the novel technique is illustrated here.

* The Field Enhancements of the Conventional Log Periodic Tooth Antenna for the different $T_{o u}$ and Alpha values are effectively demonstrated in Figures(4.1 and 4.2)

* The field enhancements of the innovative hybrid ABCPSO and the modern methods like the ABC, PSO and GA are elegantly employed with the aim of the parameter optimization.

* The novel scheme emerges as the best technique for the field enhancement of the log periodic tooth antenna. 
Table 2 Purcell Factor of Conventional Log Periodic Tooth Antenna for different Alpha, $\mathrm{N}$ and $T_{o u}$ values

\begin{tabular}{|c|c|c|c|c|}
\hline \multicolumn{5}{|c|}{$\begin{array}{l}\text { Purcell Factor of Conventional Lo } \\
\text { Tooth Antenna }\end{array}$} \\
\hline \multirow[b]{2}{*}{ Iteration } & \multirow{2}{*}{$\begin{array}{l}\text { Proposed } \\
\text { Technique } \\
\boldsymbol{A B C} \text {-PSO }\end{array}$} & \multicolumn{3}{|c|}{ Existing Methods } \\
\hline & & $\boldsymbol{G A}$ & $A B C$ & PSO \\
\hline 50 & 2.3 & 20.6 & 5.6667 & 7.8 \\
\hline 75 & 2.8 & 28.6 & 8.9 & 7.8 \\
\hline 100 & 1.6 & 14.6 & 4.9 & 5.9 \\
\hline
\end{tabular}

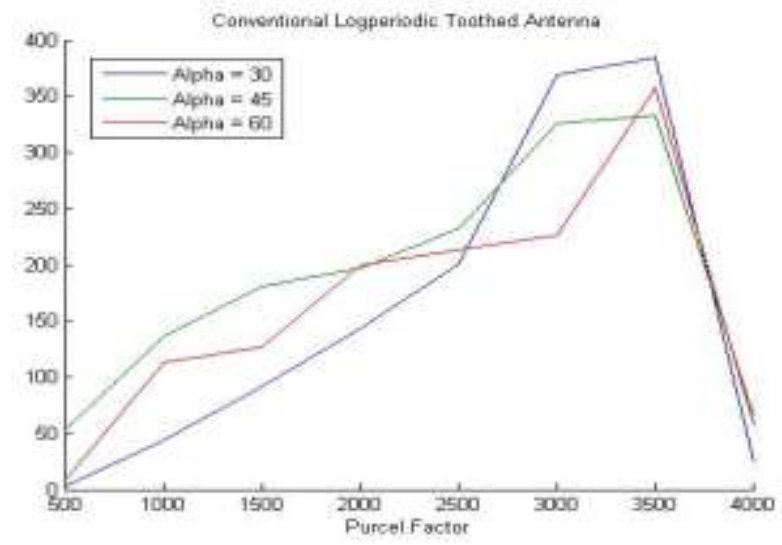

Fig 4.3 Purcell Factor of Conventional Log Periodic Tooth Antenna for Alpha=30, 45 and 60

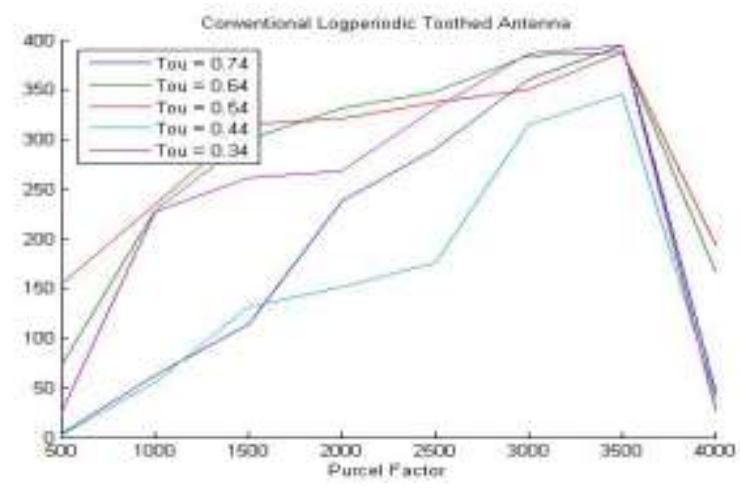

Fig 4.4 Purcell Factor of Conventional Log Periodic Tooth Antenna for different $T_{o u}$ values

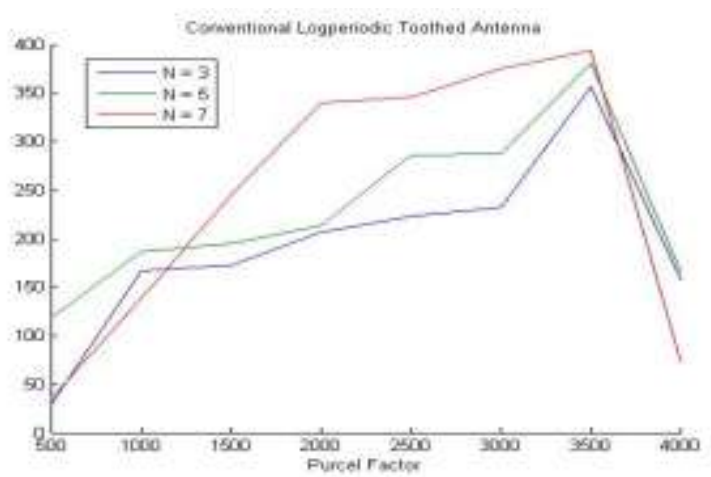

Fig 4.5 Purcell Factor of Conventional Log Periodic Tooth Antenna for different $\mathrm{N}$ values
* The Purcell factors of the Conventional Log Periodic Tooth Antenna for different Alpha, $\mathrm{N}$ and $T_{\text {ou }}$ values are brilliantly brought out in Figures (4.3-4.5)

* The Purcell factors of the new-fangled hybrid ABCPSO and the modern approaches like the ABC, PSO and GA are elegantly employed for the objective of the parameter optimization

* The novel technique turns out excellent outcome, proving its mettle as the best for the Purcell factor of the log periodic tooth antenna

* It is pertinent to note that the field enhancement and Purcell factor are somewhat sensitive to the tooth angle, whose variation triggers incredible impacts on the spectral attributes of the LPNA.

Table 3 Field Enhancement of Log periodic Toothed Circular Nano Antenna for different values

\begin{tabular}{|c|c|c|c|c|}
\hline \multicolumn{5}{|c|}{$\begin{array}{l}\text { Field Enhancement of Log periodic Toothed } \\
\text { Circular Nano Antenna }\end{array}$} \\
\hline \multirow[b]{2}{*}{ Iteration } & \multirow{2}{*}{$\begin{array}{l}\text { Proposed } \\
\text { Technique } \\
\boldsymbol{A B C} \text {-PSO }\end{array}$} & \multicolumn{3}{|c|}{ Existing Methods } \\
\hline & & $\boldsymbol{G A}$ & $A B C$ & PSO \\
\hline 50 & 28.3 & 10.9 & 10.6 & 7.85 \\
\hline 75 & 10.8 & 12.9 & 6.5 & 3.2 \\
\hline 100 & 19.7 & 20.8 & 14.8 & 3.33334 \\
\hline
\end{tabular}

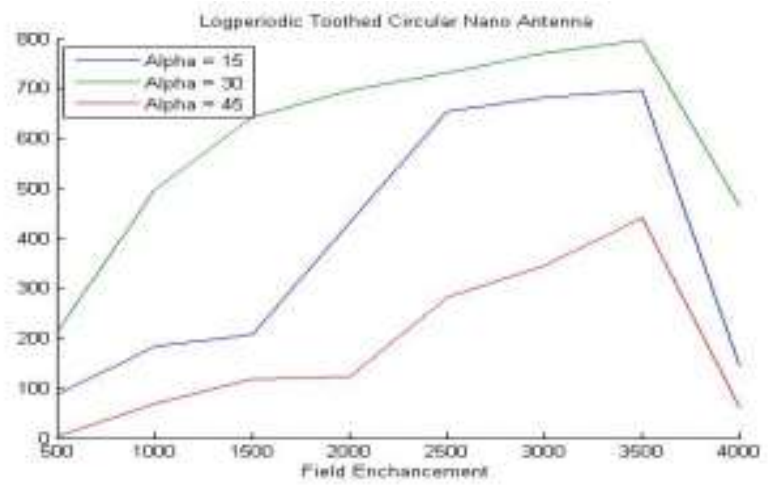

Fig 4.6 Field Enhancement of LPTCNA for Alpha=15, 30 and 45

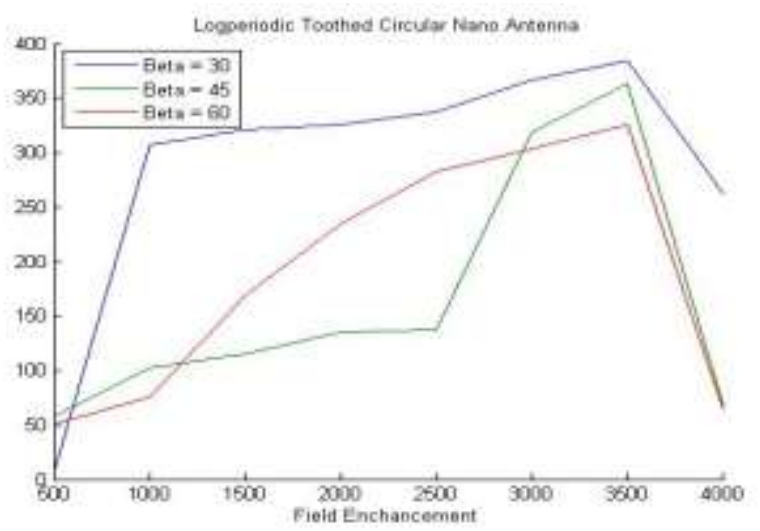

Fig 4.7 Field Enhancement of Log periodic Toothed Circular Nano Antenna for different Beta values 
* The Field enhancements of the Log periodic Toothed Circular Nano Antenna for the different Alpha, Beta and $\mathrm{N}$ values are beautifully depicted in Figures (4.6 and 4.8)

* The Field enhancements of the novel hybrid ABC-PSO and the modern approaches like the $\mathrm{ABC}$, PSO and GA are efficiently utilized for the purpose of the parameter optimization

* The suggested scheme surfaces as the significant and superlative technique, turning out the best values for the Field enhancement of LTCNA.

* The log periodic nano antenna is evaluated for both the Purcell factor and field enhancement which makes it crystal clear that the anticipated approach is incredibly impressive as the optimal output.

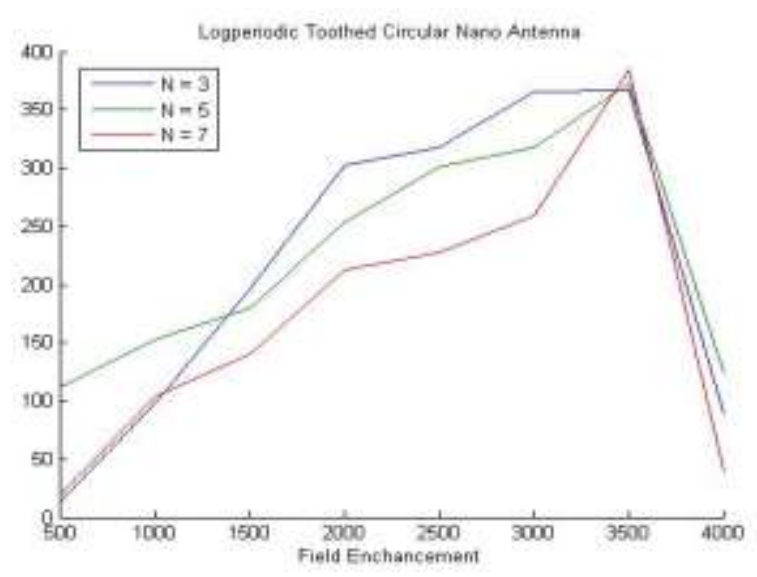

Fig 4.8 Field Enhancement of LPTCNA for different N values

Table 4 Purcell factor of Log periodic Toothed Circular Nano Antenna for different iteration

\begin{tabular}{|l|l|l|l|l|}
\hline $\begin{array}{l}\text { Purcell factor of Log periodic Toothed Circular } \\
\text { Nano Antenna }\end{array}$ \\
\hline & $\begin{array}{l}\text { Proposed } \\
\text { Technique }\end{array}$ & \multicolumn{4}{l|}{ Existing Methods } \\
\hline Iteration & ABC-PSO & GA & ABC & PSO \\
\hline 50 & 15.6 & 15.6 & 12.6 & 2.25 \\
\hline 75 & 20.9 & 13.6 & 17.6 & 6.5556 \\
\hline 100 & 14.6 & 8.7 & 5.5 & 4.1 \\
\hline
\end{tabular}

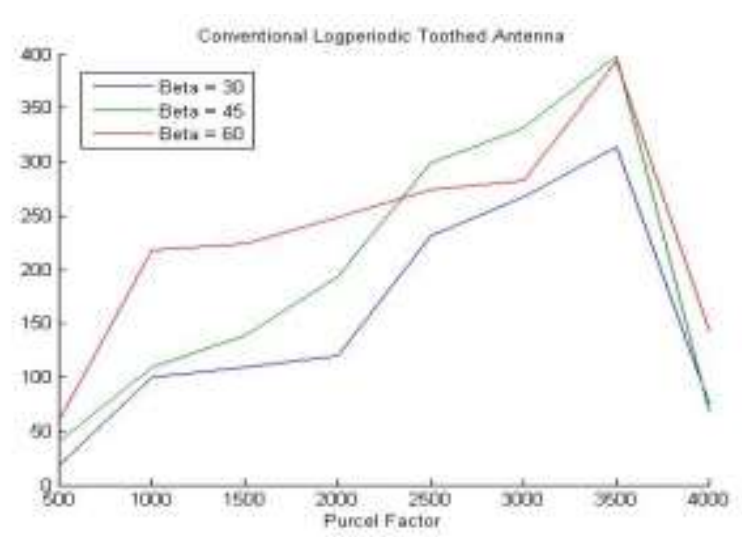

Fig 4.9: Purcell factor of Conventional Log periodic Toothed Antenna for different Beta values

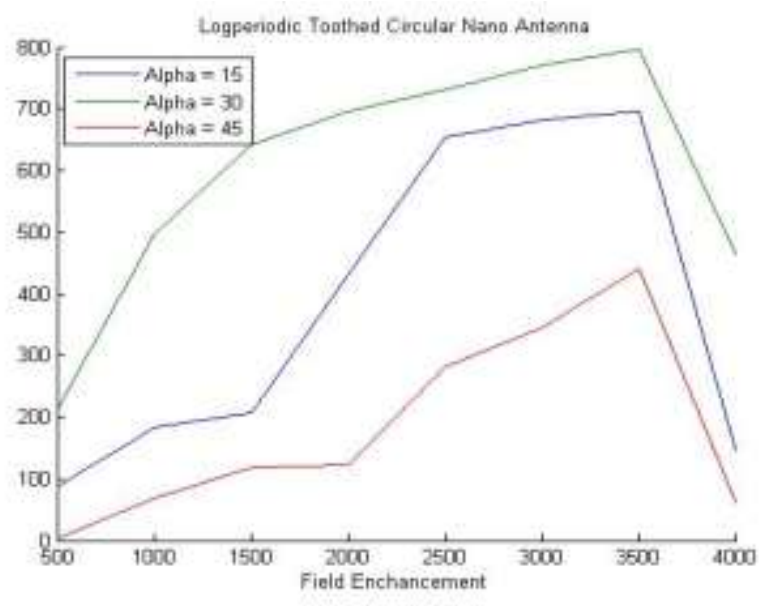

Fig 4.10 Purcell factor of Log periodic Toothed Circular Nano Antenna for different Alpha values

The outcomes for the Purcell factor and field enhancement of the LPNA have been assessed, analyzed and discussed here and the novel technique is established to be extremely effective, based on the corresponding results and debates.

* The Purcell factors of the Log periodic Toothed Circular Nano Antenna for the different Alpha and Beta values are elegantly exhibited in Figures (4.9 and 4.10).

* The Purcell factors of ground-breaking hybrid ABCPSO and the modern methods like the ABC, PSO and GA are effectively employed for the purpose of the parameter optimization

* The epoch-making technique proves its mettle and projects its par-excellence performance, realizing the best outcomes for the Purcell factor of Log periodic Toothed Circular Nano Antenna.

* A series of investigations and the consequential assessments go a long way in underscoring the amazing efficiency in performance of the dream scheme.

\section{CONCLUSION}

A single antenna to support various allotted frequency bands is difficult and sometimes results in terms of the deterioration of the signal performance. In the document, an innovative hybrid ABC-PSO optimization approach is envisioned dedicated for the design and optimization of the $\log$ periodic nano-antenna parameters. The underlying objective of the novel technique is invested in the incredible improvement in the efficiency of the design parameter. With an eye on designing the log periodic nano antenna by adapting the parameter which is well-geared for the wireless communication is estimated for the field improvement and Purcell factor of Conventional Log periodic Toothed Antenna and Log periodic Toothed Circular Nano Antenna.

The technique has achieved better results in terms of both the Purcell factor and the field enhancement of the conventional log periodic nano antenna and the log periodic toothed circular nano antennas. Moreover, the analysis is compared with the recently developed optimization techniques so as to prove the efficiency of our proposed technique. The eye-catching test outcomes illustrate the fact 
without an iota of ambiguity that our masterpiece method flies high in the sky as the most excellent and effective system vis-a-vis the modern techniques like the ABC, PSO and genetic algorithm.

The comparison made graphically could make us to understand easily, regarding the attainment of better outcome while varying the target parameters. This dynamically shows us how to get better performance. This also tells us that, on what design parameters the antenna must be tuned.

\section{REFERENCES}

[1]. H. F. Abutarboush, and A. Shamim, "Paper-Based Inkjet-Printed Tri-Band U-Slot Monopole Antenna for Wireless Applications", IEEE, Vol. 11, pp. 1234-1237, 2012.

[2]. I. Wang and Y. Du, "Directional field enhancement of dielectric nano optical disc antenna arrays", Vol. 34, No. 1, pp. 126-130, 2011.

[3]. M. N. Cia and S. A. Maier, "Broad-Band Near-Infrared Plasmonic Nano antennas for Higher Harmonic Generation”, Vol. 6, No. 4, pp. 3537-3544, 2012.

[4]. D. K. Kotter, S. D. Novack ,W. D. Slafer, and P. Pinhero, "Solar Nantenna Electromagnetic Collectors", American Society of Mechanical Engineers,Idaho National Laboratory, pp. 409-415, 2008.

[5]. D. M. Solísa, J. M. Taboada, M. G. Araújoa, F. Obelleiroa, and J. O. López, "Design of optical wide-band log-periodic nano antennas using surface integral equation techniques", Vol. 301, pp. 61-66, 2013.

[6]. G. Zhai, Y. Cheng, Q. Yin, L. Chiu, S. Zhu, and J. Gao, "Super High Gain Substrate Integrated Clamped-Mode Printed Log-Periodic Dipole Array Antenna", Vol. 61, No. 6, pp. 3009-3016, 2013.

[7]. Z. S. Hamidi, N. N. M. Shariff, "Evaluation of signal to noise ratio (SNR) of log periodic dipole antenna (LPDA)", Business Engineering and Industrial Applications Colloquium (BEIAC), IEEE, pp. 434-438, 2013.

[8]. D. R. Ward, F. Pauly, J. C. Cuevas and D. Natelson., "Optical rectification and field enhancement in a plasmonic nanogap", Nature Nanotechnology, Vol. 5, No. 10, pp. 732-736, 2010.

[9]. F. Carreñon, F. A. Yáñez, and M. A. Antón, "Spin initialization of a p-doped quantum dot coupled to a bowtie nano antenna", Vol. 343, pp. 97-106, 2015.

[10]. S. Panaro, Toma, P. Zaccaria, Chirumamilla, Saeed, Razzari, Liberale, D. Angelis, and Fabrizio, "Design and top-down fabrication of metallic L-shape gap nanoantennas supporting plasmon-polariton modes", Vol. 111, pp.91-95, 2013.

[11].S. Jafarlou, M. Neshat, S. S. Naeini, “A Fast Method for Analysis of Guided Waves and Radiation from a NanoScale Slit Loaded Waveguide for a $\mathrm{THz}$ Photoconductive Source", Vol. 2, No. 6, pp. 652-658, 2012.

[12]. M. C. Marcon, "Nano-Structuring and NanoFabrication Enabled by Extreme Ultraviolet Laser Sources", Vol. 5, No. 2, 2013.

[13]. G. Zhai, Y. Cheng, Q. Yin, and S. Zhu, "Gain Enhancement of Printed Log-Periodic Dipole Array
Antenna Using Director Cell", Vol. 62, No. 11, pp. 59155919, 2014.

[14].S. Wang, Y. Gong, Y. Hou, Z. Wang, "Study of a LogPeriodic Slow Wave Structure for Ka-band Radial Sheet Beam Traveling Wave Tube", Vol. 41, No. 8, pp. 22772282, 2013.

[15]. A. E. Miroshnichenko, I. S. Maksymov, A. R. Davoyan, C. Simovski, P. Belov, and Y. S. Kivshar, "An arrayed nanoantenna for broadband light emission and detection”, Vol. 5, No. 9, pp. 347-349, 2011.

\section{BIOGRAPHIES}

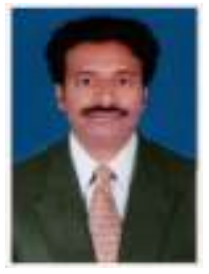

SATHISHA THIMMAIAH obtained his Bachelor's degree in Electronics and Communication from University of Mysore. Then he obtained his Master's degree in Digital Communication and Networking and Pursuing $\mathrm{PhD}$ in Electronics majoring in Nano Antenna Currently, he is an Asst Professor at the Faculty of Electrical and Electronics, Ghousia College Engineering, Ramanagara.

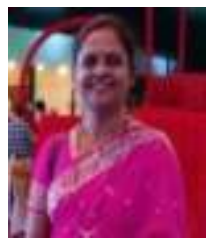

Dr.R.AnandaKumari received her M.Sc degree in physics from Mysore University, M.Phil and Ph.D from Bangalore University. She is working as an Associate Professor and Head of Department of Physics in Sree Siddaganga College of Arts, Science and Commerce for Women, Tumkur Since 1983. She is serving as the IQAC coordinator of the college. She has the Teaching experience of about 32 years \& research experience of 20 years. Her areas of research interest are Growth and Characterization of Mixed Alkali halide Crystals, Synthesis and Characterization of Nano phosphors \& Growth and Characterization of NLO materials. 